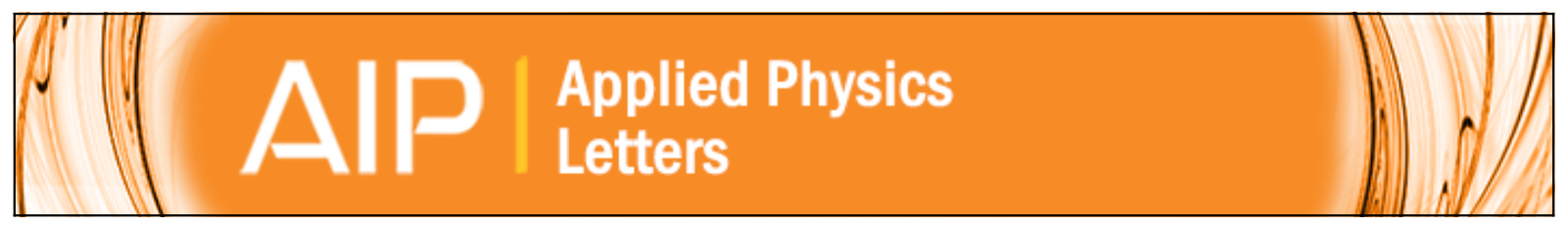

Determination of single-pass optical gain and internal loss using a multisection device J. D. Thomson, H. D. Summers, P. J. Hulyer, P. M. Smowton, and P. Blood

Citation: Applied Physics Letters 75, 2527 (1999); doi: 10.1063/1.125066

View online: http://dx.doi.org/10.1063/1.125066

View Table of Contents: http://scitation.aip.org/content/aip/journal/apl/75/17?ver=pdfcov

Published by the AIP Publishing 


\title{
Determination of single-pass optical gain and internal loss using a multisection device
}

\author{
J. D. Thomson, ${ }^{\text {a) }}$ H. D. Summers, P. J. Hulyer, P. M. Smowton, and P. Blood \\ Department of Physics and Astronomy, University of Wales Cardiff, Cardiff, CF2 3YB, United Kingdom
}

(Received 13 July 1999; accepted for publication 30 August 1999)

\begin{abstract}
We describe a technique for the measurement of optical gain and loss in semiconductor lasers using a single, multisection device. The method provides a complete description of the gain spectrum in absolute units and over a wide current range. Comparison of the transverse electric and transverse magnetic polarized spectra also provides the quasi-Fermi-level energy separation. Measurements on AlGaInP quantum well laser structures with emission wavelengths close to $670 \mathrm{~nm}$ show an internal loss of $10 \mathrm{~cm}^{-1}$ and peak gain values up to $4000 \mathrm{~cm}^{-1}$ for current densities up to $4 \mathrm{kA} \mathrm{cm}^{-2}$. (C) 1999 American Institute of Physics. [S0003-6951(99)04243-6]
\end{abstract}

To assess the performance of a semiconductor laser, knowledge of the gain and loss at a known pumping level is essential. The optical gain can be determined either by looking at the longitudinal modes in the stimulated emission (Hakki-Paoli technique), ${ }^{1}$ by transforming the spontaneous emission (Henry technique), 2,3 or by analyzing the amplified spontaneous emission as a function of length. ${ }^{4}$ A drawback of the Hakki-Paoli technique is that a high spectral resolution is required to resolve the longitudinal modes, ${ }^{5}$ while the Henry technique uses an indirect method to calculate the Fermi-level energy separation and does not directly give gain in absolute units but can be calibrated. Furthermore, both techniques are limited to current densities below the threshold of the device. Oster et al. have analyzed the amplified spontaneous emission (ASE) using a variable stripe method, where different contact stripe lengths were deposited onto the sample and the ASE of each stripe measured. However, this requires a constant collection efficiency. Techniques using segmented devices have also been used to obtain gain spectra. ${ }^{6,7}$ Optical gain spectra that use the variable stripe length method ${ }^{8,9}$ or transmission experiments, ${ }^{10}$ and derived by optical gain spectroscopy, are also available.

Although the mirror loss can be calculated using the Fresnel equations, the total loss is difficult to ascertain. The internal loss can be measured through the external differential efficiency variation as a function of the device length above threshold, however, it is difficult to obtain accurate data. ${ }^{11}$ Alternatively, the mode loss can be measured by detecting the attenuation of optically induced luminescence, generated by an external pump laser as a function of the distance of the excitation from the sample edge. ${ }^{12}$ This method requires careful implementation and relies on having an appropriate pump source.

In this letter, we show how the optical gain and the internal loss can be determined with the use of a single multisection device, as illustrated in Fig. 1. There are five, 300$\mu \mathrm{m}$-long sections which have separate contacts for electrical excitation. To obtain gain, we measured the amplified spontaneous emission when pumping section one; sections one

${ }^{a)}$ Electronic mail: thomsonjd@cf.ac.uk and two; sections one, two, and three; and so on, thus increasing the length of the active cavity. For the loss measurement, we pump each single section in sequence to increase the passive length. The gain measurement depends upon measuring a single pass, and to ensure this, we use a 600$\mu \mathrm{m}$-long absorber and at one end an angled facet. Additionally, current densities above the typical threshold of a laser device are made available.

We have measured the facet emission from a $50-\mu \mathrm{m}-$ wide stripe, with a single compressively strained 68 - $\AA$-wide $\mathrm{Ga}_{0.41} \mathrm{In}_{0.59} \mathrm{P}$ quantum well within an $\left(\mathrm{Al}_{0.5} \mathrm{Ga}_{0.5}\right)_{0.51} \mathrm{In}_{0.49} \mathrm{P}$ waveguide core and $\left(\mathrm{Al}_{0.7} \mathrm{Ga}_{0.3}\right)_{0.51} \mathrm{In}_{0.49} \mathrm{P}$ cladding. This structure was grown on a (100)-axis, GaAs substrate that has a $10^{\circ}$ misorientation to $(111 \mathrm{~A})$, which is used to make the angled facet. At $300 \mathrm{~K}$, a 320- $\mu \mathrm{m}$-long laser made with the same material emits a wavelength of $(671.3 \pm 1.0) \mathrm{nm}$. A $4-\mu \mathrm{m}$-wide trench is etched between the section to provide current confinement. We etched through the GaAs cap layer only, to avoid introducing extra mode loss. This is not sufficient to totally electrically isolate the sections, and so, some degree of current spreading occurs. Therefore, to ensure that the same current density is applied to different lengths, the spontaneous emission intensity through a top window in the device is measured and is used as an indicator of the radiative recombination rate.

To obtain the internal loss, we pump sections to act as individual sources of ASE ensuring the same current density.

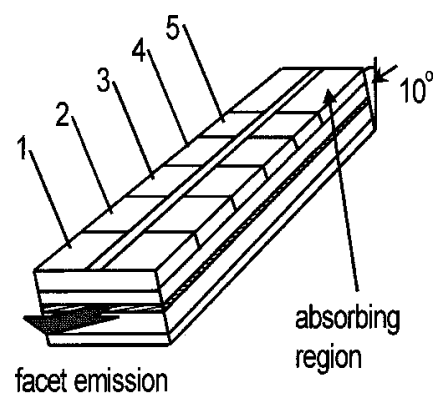

FIG. 1. Schematic of the multisection device for gain and loss measurements. The front five sections are for electrical injection and the absorbing region and angled facet is to ensure the single-pass nature of the measurement. 


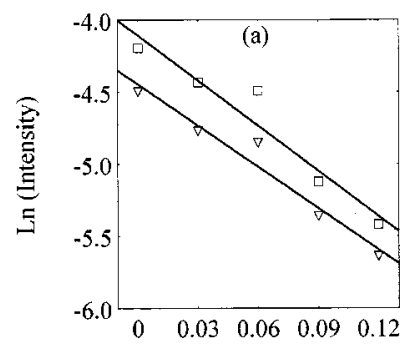

Transmission Length (cm)

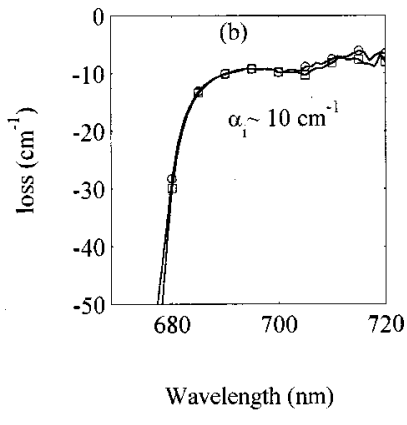

FIG. 2. (a) Logarithmic plot of intensity vs passive transmission length at wavelengths of $690 \mathrm{~nm}$ (squares) and $692 \mathrm{~nm}$ (triangles). (b) Spectral measurement of loss for $900 \mu \mathrm{m}$ (squares) and $1200 \mu \mathrm{m}$ (circles) passive lengths.

Due to band-gap renormalization in a pumped section, emission occurs at energies below the absorption edge of the unpumped regions. This emission at lower energies is guided along the device, attenuated only by internal scattering. The transmitted intensity $I_{t}$ is related to the initial ASE intensity $I$ and the transmission length $L$, by the following equation:

$$
I_{t}=I\left(e^{-\alpha_{i} L}\right),
$$

where $\alpha_{i}$ is the internal loss coefficient. Figure 2(a) shows the measured transmitted intensity on a log scale plotted against the length of the passive device as described by Eq. (1). A linear relationship occurs, as expected; the gradient gives us the internal loss coefficient. We calculate a value for $\alpha_{i}$ of $10 \pm 1 \mathrm{~cm}^{-1}$ for this device using a range of different wavelengths. The transmission length in Fig. 2(a) is taken to be the physical length of the contact sections (i.e., multiples of $300 \mu \mathrm{m})$. However, the actual length of the passive section will be reduced by a fixed amount due to current spreading. The current spreading in each section is assumed to be the same, which does not seem unreasonable. This manifests itself as a rigid shift in the $x$ axis giving the same value for the gradient, and hence, not affecting the measured value of $\alpha_{i}$. As the optical source is broadband spontaneous emission, the attenuated transmission can be measured over a wide wavelength range. Therefore, we are able to spectrally resolve the internal loss coefficient. Figure 2(b) shows the loss spectrum for 900 and $1200 \mu \mathrm{m}$ passive lengths. In the short-wavelength region we see the absorption edge of the quantum well dominating the curve. It then flattens out to give a value of $\sim 10 \mathrm{~cm}^{-1}$ over the region in which the optical attenuation is due only to scattering and free-carrier absorption losses. It can be seen that these spectrally resolved values, obtained from the comparison of any two lengths, agree with the values obtained from the full plot in Fig. 2(a).

To obtain the gain, we pump a series of sections together to vary the length of the active stripe. The ASE intensity is measured through a polarizer allowing both transverse electric (TE) and transverse magnetic (TM) polarized gain to be calculated. By integration the expression for the amplification of light from a point source, a distance $L$ from the facet, over the length of the device, the net modal gain $G$ can be related to the ASE intensity $I$ by ${ }^{4}$

$$
I=\frac{I_{\mathrm{sp}}}{G}\left(e^{G L-1}\right),
$$

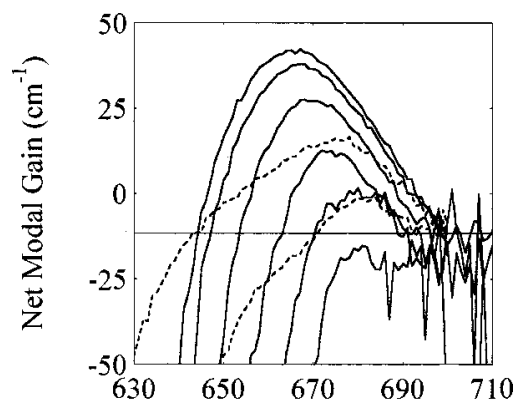

\section{Wavelength (nm)}

FIG. 3. Plot of the measured TE (solid lines) and TM (dashed lines) gain spectra at current densities of 3.0, 2.6, 2.0, 1.3, 1.0, and $0.6 \mathrm{kA} \mathrm{cm}^{-2}$ for the TE spectra and 3.0 and $1.0 \mathrm{kA} \mathrm{cm}^{-2}$ for the TM spectra. The horizontal line shows the value of $\alpha_{i}$ from the crossover of TE and TM spectra.

where $I_{\mathrm{sp}}$ is the intensity of the spontaneous emission. The net modal gain $G$ is related to the material gain $g$ and the internal loss $\alpha_{i}$ by

$$
G=\Gamma g-\alpha_{i},
$$

where $\Gamma$ is the optical confinement factor. Equation (2) does not give a direct method to obtain the gain; however we can compare the ASE intensity spectra for different lengths to obtain an analytical expression for the gain $G$. The intensity from device lengths of $2 L\left(I_{2 L}\right)$ and $L\left(I_{L}\right)$ were compared to obtain the following expression for the gain $G$ :

$$
G=\frac{1}{L}\left[\ln \left(\frac{I_{2 L}}{I_{L}}-1\right)\right]
$$

This allows us simply to obtain the gain spectra in absolute units directly for the recorded ASE intensity. Figure 3 shows the net modal TE and TM gain for different current densities at room temperature. The quasi-Fermi-level energy separation between the electrons and holes is equal to the photon energy at which the material gain $g$ is zero, i.e., the transparency point. As the population of carriers in the heavy- and light-hole bands are described by the same Fermi function, the TE and TM gain spectra cross at the transparency point, and hence, this crossover provides a direct measurement of the quasi-Fermi-level energy separation. At this point, Eq. (3) becomes $G=-\alpha_{i}$, and from Fig. $3 \alpha_{i}$ is determined to be $13 \pm 2 \mathrm{~cm}^{-1}$ for different current densities. This assumes that TE and TM polarizations have the same internal loss.

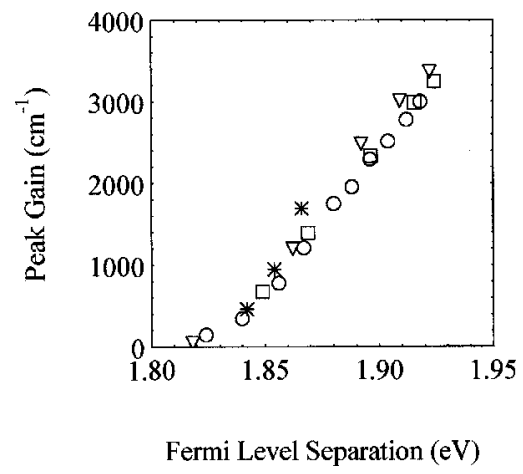

FIG. 4. Peak gain vs quasi-Fermi-level energy separation. Different symbols represent three separate devices while the star symbols represent previous data values (Ref. 13). 
The values for $\alpha_{i}$ agree will with the value found using the transmission experiment described earlier. In Fig. 4 we have plotted the peak material gain against the Fermi-level energy separation for three different devices from the same material, where we have converted the modal gain into material gain using Eq. (3) and a calculated confinement factor of $1.6 \%$. The points lie on the same curve, demonstrating consistency of the measurement technique when applied to a number of devices. Figure 4 additionally shows values determined from previous experimental and theoretical studies ${ }^{13}$ and are in agreement.

In summary, we have developed a technique for measuring the optical gain and loss using a single multisection device. The key benefits of the method are that it does not require high spectral resolution as it is based upon measurement of the amplification of spontaneous emission and that it provides, directly, the gain in absolute units and the quasiFermi-level energy separation. The method also provides an independent measurement of the internal loss via lengthdependent transmission experiments.

The authors acknowledge the financial support of the
Engineering and Physical Sciences Research Council for a studentship for one of the authors (J.D.T.) and a Research Fellowship for one of the authors (H.D.S.).

${ }^{1}$ B. W. Hakki and T. L. Paoli, J. Appl. Phys. 46, 1299 (1975).

${ }^{2}$ C. H. Henry, R. A. Logan, and F. R. Merritt, J. Appl. Phys. 51, 3042 (1980).

${ }^{3}$ P. Blood, A. I. Kucharska, J. P. Jacobs, and K. Griffiths, J. Appl. Phys. 70, 1144 (1991).

${ }^{4}$ A. Oster, G. Erbert, and H. Wenzel, Electron. Lett. 33, 864 (1997).

${ }^{5}$ L. A. Lam Sin Cho, P. M. Smowton, and B. Thomas, Proc. IEEE 137, 64 (1990).

${ }^{6}$ S. D. McDougall and C. N. Ironside, Electron. Lett. 31, 2179 (1995).

${ }^{7}$ F. C. Prince, T. J. S. Mattos, and N. B. Patel, Electron. Lett. 18, 1055 (1982).

${ }^{8}$ K. L. Shaklee and R. F. Leheny, Appl. Phys. Lett. 18, 475 (1971).

${ }^{9}$ E. Zielinski, F. Keppler, S. Hausser, M. H. Pilkuhn, R. Sauer, and W. T. Tsang, IEEE J. Quantum Electron. QE-25, 1407 (1989).

${ }^{10}$ C. Ellmers, M. Hofmann, W. W. Ruhle, A. Girndt, F. Jahnke, W. W. Chow, A. Knorr, S. W. Koch, C. Hanke, L. Korte, and C. Hoyler, Phys. Status Solidi B 206, 407 (1998).

${ }^{11}$ P. M. Smowton and P. Blood, Appl. Phys. Lett. 70, 2365 (1997).

${ }^{12}$ P. C. Mogensen, P. M. Smowton, and P. Blood, Appl. Phys. Lett. 71, 1975 (1997).

${ }^{13}$ W. W. Chow, P. M. Smowton, P. Blood, A. Girndt, F. Janke, and S. W. Koch, Appl. Phys. Lett. 71, 157 (1997). 